This is the authors' final version, post peer-review, of an article published in BMJ Quality \& Safety 2013;22(2):97-102. The definitive version is available from http://qualitysafety.bmi.com/

\title{
Perceived causes of prescribing errors by junior doctors in hospital in- patients: A study from the PROTECT programme.
}

Dr. Sarah Ross, Senior Lecturer, Division of Medical and Dental Education, School of Medicine and Dentistry, University of Aberdeen

Dr. Cristín Ryan, Lecturer in Clinical Pharmacy, School of Pharmacy, Queen's University Belfast

Dr. Eilidh M Duncan, Research Fellow, Health Psychology Group, University of Aberdeen

Professor Jill J Francis, Professor of Health Psychology, Health Psychology Group, University of Aberdeen

Professor Marie Johnston, Professor of Health Psychology, Health Psychology Group, University of Aberdeen

Professor Jean Ker, Professor of Medical Education, School of Medicine, University of Dundee Professor Amanda Jane Lee, Professor of Medical Statistics, Medical Statistics Team, University of Aberdeen

Dr. Mary Joan MacLeod, Senior Lecturer, School of Medicine and Dentistry, University of Aberdeen Professor Simon Maxwell, Professor of Student Learning/Prescribing, Clinical Pharmacology Unit, University of Edinburgh, Western General Hospital, Edinburgh

Dr. Gerard McKay, Consultant Physician, University Medical Unit, Glasgow Royal Infirmary

Dr. James McLay, Senior Lecturer, School of Medicine and Dentistry, University of Aberdeen

Professor David J Webb, Christison Professor of Therapeutics and Clinical Pharmacology, Clinical Pharmacology Unit, University of Edinburgh, Royal Infirmary of Edinburgh

Professor Christine Bond, Professor of Primary Care and Pharmacy, Centre of Academic Primary Care, Division of Applied Health Sciences, University of Aberdeen

\section{Corresponding author:}

Dr. Sarah Ross 
Senior Lecturer

Division of Medical and Dental Education

School of Medicine and Dentistry

University of Aberdeen

Room 2:036 Polwarth Building

Foresterhill

Aberdeen

tel 01224437899

fax 01224437921

emails.ross@abdn.ac.uk

Running Title: Causes of prescribing errors

Keywords: junior doctors, prescribing, errors, causes, human error theory, patient safety

Word count: 3292

Number of Tables: 2

Number of Figures: 0 


\section{Abstract}

\section{Introduction}

Prescribing errors are a major cause of patient safety incidents. Understanding the underlying factors is essential in developing interventions to address this problem.

This study aimed to investigate the perceived causes of prescribing errors among foundation (junior) doctors in Scotland.

\section{Methods}

In eight Scottish hospitals, data on prescribing errors were collected by ward pharmacists over a 14-month period. Foundation doctors responsible for making a prescribing error were interviewed about the perceived causes. Interview transcripts were analysed using content analysis and categorised into themes previously identified under Reason's Model of Accident Causation and Human Error.

\section{Results}

Forty prescribers were interviewed about 100 specific errors. Multiple perceived causes for all types of error were identified and were categorised into five categories of error producing conditions, (environment, team, individual, task and patient factors). Work environment was identified as an important aspect by all doctors, especially workload and time pressures. Team factors included multiple individuals and teams involved with a patient, poor communication, poor medicines reconciliation and documentation and following incorrect instructions from other members of the team. A further team factor was the assumption that another member of the team would identify any errors made. The most frequently noted individual factors were lack of personal knowledge and experience. The main task factor identified was poor availability of drug information at admission and the most frequently stated patient factor was complexity.

\section{Conclusions}

This study has emphasised the complex nature of prescribing errors, and the wide range of error-producing conditions within hospitals including the work environment, team, task, individual and patient. Further work is now needed to develop and assess interventions that address these possible causes in order to reduce prescribing error rates. 


\section{Introduction}

Patient safety remains a substantial challenge to healthcare systems, with medication related events accounting for a consistently reported high number of all adverse events (13). The Audit Commission reported that 1100 patients died in the UK during 2001 from medication errors or adverse drug reactions, while others had extended in-patient hospital stays (1). The National Patient Safety Agency in 2007 received 92 reports of serious harm or death from medication errors (2). Prescribing errors are known to account for a substantial proportion of medication errors and cause the most significant problems (4). Junior doctors are the most frequent prescribers in the hospital setting and make most of the prescribing errors $(5,6)$. The promotion of safe medication use and the reduction of iatrogenic harm are of vital importance, as demonstrated by initiatives such as the Scottish Patient Safety Programme (7). An understanding of the underlying factors is an essential step in developing strategies to address this problem.

A range of possible causes for errors have been reported (8) but relatively few studies have specifically investigated junior doctors' prescribing. A systematic review of the literature (10) identified 16 studies and then analysed them using Reason's Human Error Theory (11). Reported errors were found to be mainly mistakes (i.e. an intended action which goes wrong), with error-provoking conditions of stress, fatigue, high workload, lack of experience or training and poor communication noted. Dornan et al. highlighted similar issues (5). Dean et al. reported themes such as lack of feedback, lack of documentation of prescribing decisions, a focus on the drug but not dose and frequency, poor quality of medication information on hospital admission and a lack of willingness by doctors to challenge senior decisions (8).

The PROTECT ('PRescribing Outcomes for Trainee doctors Engaged in Clinical Training') study was a programme of work to investigate the prevalence and causes of prescribing errors specifically among doctors in the first two years of their postgraduate training (foundation year 1 (F1) and 2 (F2)) to compare these with those of other hospital doctors, and to describe F1 and F2 doctors' knowledge of and attitudes towards prescribing errors, in Scotland. This paper reports on the results of interviews undertaken with junior doctors who had made a prescribing error during the study period.

\section{Methods}

One teaching hospital and one district general hospital in each of the four post graduate deaneries in Scotland were invited to participate in the study. Hospitals meeting the inclusion criteria (at least $12 \mathrm{~F} 1 \mathrm{~s}$ working in the hospital) were approached sequentially via the Health Board Director of Pharmacy and the Chief Hospital Pharmacist, until target recruitment was met. In one area, two teaching hospitals divided the data collection 
between them, giving a total of nine hospitals. Consent was obtained from both the Director of Pharmacy and the Medical Director at each hospital.

In each hospital, data on prescribing errors (defined by Dean (9)) were collected by ward pharmacists for one week from two wards per calendar month over a 14-month period (equating to a total of 112 ward weeks). Wards were purposively selected to ensure a range of adult medical, surgical, acute and long stay wards were included.

F1 and F2 doctors responsible for making a prescribing error were approached by their ward clinical pharmacist within 96 hours of writing the prescription and were invited to participate in a semi-structured interview with the lead researcher (CR). The pharmacist provided contact details of those consenting to the researcher, who made the interview arrangements with the prescriber. Where possible, the interview was conducted within the 96 hour window. The interview schedule included questions about the error type, circumstances surrounding the error, perceived causes and potential consequences had the error not been intercepted by the pharmacist. Interviews were conducted either face to face, or by telephone and were recorded and transcribed verbatim. To maximise honest discussion in the interviews, confidentiality was assured; the interviewer was an academic pharmacist with an understanding of the possible issues relating to the occurrence of prescribing errors, yet independent of any of the NHS organisations.

Errors were subdivided into types according to a classification system derived from the literature and our previous work $(8,9)$ (Table 1$)$.

Transcripts were analysed using content analysis, the most appropriate approach when themes of interest are predetermined. These themes were previously identified under Reason's Model of Accident Causation (10) which considers latent (organisational (e.g. workload) and management (eg staffing levels, culture) and error-producing conditions (environment, task, team, individual and patient). Errors were also further classified using this model into the types of errors that occurred (slips, lapses, mistakes and violations). This theory was chosen as a well used model in patient safety which combines both environment and individual factor. The analysis was undertaken separately by two researchers (CR, SR), who then met to compare and agree on themes identified. Any disagreements were resolved by discussion.

The study was approved by the North East of Scotland Research Ethics Committee, and NHS $\mathrm{R}$ and $\mathrm{D}$.

\section{Results}

Pharmacists provided contact details for 54 doctors. All 54 were contacted, but due to work schedules, only 40 prescribers were interviewed regarding 100 specific errors (the maximum number of errors discussed in an individual interview was 16 which were all for one patient; the majority referred to two or three errors). No other reasons for non-participation were 
provided. The majority of interviews took place within 96 hours of the error(s) occurring. Of the doctors interviewed, 17 were female and 23 were male; 31 were F1 and 9 were F2 doctors. Doctors worked in a range of specialities. Geographically, interviewees were recruited from all four deaneries within Scotland; 12 from the North, 8 from the East, 12 from the South and 8 from the West. Fourteen interviews were conducted face to face, and 26 by telephone. Interviews were conducted on all error types (Tables $1 \& 2$ ). The most frequent errors were slips $(n=30)$, and violations were uncommon $(n=6)$. Approximately one third $(n=35)$ were unclassifiable from the information provided or were errors due to receiving poor quality information which could not be attributed to a slip, lapse, mistake or violation on the part of this individual prescriber (we did not have data to analyse what type of error the original prescriber made).

Multiple perceived causes, from all of Reason's model categories (work environment, task, team, individual and patient), were identified by foundation doctors for each category of error. These are reported below with supporting quotes identified by interviewee number.

\section{Work environment}

Work environment was identified as an important aspect by all doctors. Within this, workload and time pressures were the most commonly mentioned factors.

"for example today we're receiving, we've got nine names on the board to come in, we've got probably about, say 40/50 patients already under our care and I think that pretty much sums it up, the kind of environment that we're working in, so you could definitely put it down to that." (028)

In particular, the pressures of time were thought to impact on checking practices, whether these were gathering more information for medicines reconciliation, or checking reference texts such as the British National Formulary. Doctors also noted that they had little time to go back to a task which they had not completed.

"I left it blank, I was meant to come back and do that later but then I got caught up with doing other jobs." (024)

Many doctors reported that they were pressurised by other staff to complete discharge prescriptions, often doing several at a time and not always for patients that they knew.

"I remember it was on a ward round where you would discharge quite a few patients and I'd started to do discharge prescriptions for a few patients and then l'd obviously got two of them mixed up at the end." (009)

"A lot of the time we're really pressurised by staff to do discharge letters very quickly ... with staff actually coming to knock on our door more than three to four times while completing 
discharges, we have to finish things very quickly, finish things yesterday and it's, it becomes almost impossible." (010)

Interruptions were noted, as was the tendency to "multi-task", particularly during ward rounds.

"But then it becomes a problem, that you're sat in the doctors' room and you get this constant flow of voices and phone call and bleeps and one thing and another. You know, you're just getting constantly interrupted..." (021)

Task factors

The main task factor identified was poor availability of drug information at admission (often out of hours). Systems which should aid prescribers were not always available (e.g. the emergency care summary (an out of hours NHS health record for patients) was available, but the doctor did not have a password to access it).

"But I think the problem is that after 5 o'clock I don't have access to anything to tell me what GPs are prescribing, I don't have any emergency care summaries." (012)

Team factors

A number of important team factors were discussed. The number of different individuals and teams who were involved in a single patient journey was reported as an important cause of error. Poor communication within and between these teams was thought to contribute to error, such as when medicines reconciliation was incomplete but not handed over as a task for the next team. Similarly, fragmentation of tasks across the team was reported to add to poor shared awareness about prescriptions.

"I just did the other bits of paperwork and things, so I did her kardex and I did the other practical procedures, so I never actually took the history from her, along with her medication history, I just came in and did all the other stuff" (038)

Poor completion of tasks by the previous doctor, such as medicines reconciliation and documentation, were also blamed for error. This was often reported in the admissions setting, with doctors on subsequent wards poorly informed about medicines related decisions.

"The medical reconciliation sheet, as per usual, was not filled by the FY2 who clerks the patients in ARU (acute receiving unit), so the patient came in without that being filled..." (027) 
A further team factor was the assumption that another member of the team (most commonly the ward pharmacist) would identify errors made. Foundation doctors relied on all prescriptions being checked by another professional.

"That's probably not brilliant but I suppose like stuff like that, I know someone else is going to check it, whether it's the pharmacist or whether, I know that somebody will go through it the next morning and confirm..." (013)

Supervision was rarely mentioned; however prescribers did say that at times errors were made as a result of following instructions from other staff.

"People frequently come to you with bits of paper "You've got to do this, can you sign it?" "Yeah, that's fine, it seems like a reasonable thing to do". So you trust a lot of the nursing staff." (013)

Individual factors

The most frequently noted individual factors were lack of personal knowledge and experience.

"And that was pure, purely ignorance on my part, I didn't realise that you needed a nitrate free period, so that was my own mistake there, completely, and a lack of knowledge on my part." (006)

"I think my ignorance is a big factor because you know rather than knowing that was already a medicine that they could have been on, me just thinking "Oh gosh maybe they spelt it wrong". You know, if I'd been more aware of a wider range of drugs I might not have made the mistake." (011)

Some doctors reported attitudes towards prescribing that could be unhelpful, for example, that transcribing medicines is a low importance task, and that juniors should not change prescriptions made by other staff.

"...when you re-write these, even though it's technically, it is your prescription, often you don't alter the medicines because sometimes you don't even know the patient, you're just asked to re-write a kardex, so it's not really your place to be altering things..." (007)

"With the tramadol I probably would just have presumed that it was OK to prescribe it if it was already on the kardex. I'm maybe loathe to... I maybe don't doubt other people's prescribing, I maybe should do that a bit more, be a bit more critical about it if I'm putting my name to it on a discharge letter." (026)

The most frequently stated patient factor was complexity (e.g. polypharmacy). 
"I think most errors do tend to be when patients come into hospital and they are on a lot of drugs and there is difficulty in clarifying it." (002)

\section{Patient factors}

Poor information from patients was also noted, as was misplaced trust that the patient was giving accurate information.

" appeared to me from dealing with her before that she was a pretty clued up patient, ... she seemed to know which medications she was taking when, she was pretty aware...so I asked her what her usual regimen of MST (morphine sulphate tablets) is and that's what I gave her, and apparently that wasn't correct." (027)

\section{Discussion}

The PROTECT programme of work is being undertaken to investigate prescribing errors in Scottish hospitals. This qualitative phase of the work suggests a range of latent factors and error-provoking conditions in the workplace are implicated when foundation grade doctors make errors. Conditions could be categorised using Reason's model (12). Work environment factors were most commonly cited, although a range of other factors, such as individual lack of knowledge were also mentioned.

As in previous studies, problems with inadequate quality medicines information at admission to hospital were highlighted. It is disappointing to see that measures such as the Emergency Care Summary (ECS) which have been designed to tackle this very issue by providing an up to date list of patient's medicines are not working (many doctors said that they did not have access to ECS). Medicines reconciliation, a process by which the most recent and accurate sources of information are used to create a full list of medicines for a patient, has been a focus of efforts in many Scottish hospitals. However despite this focus of effort, doctors reported that this process and its documentation were poorly used. Discharge prescriptions were considered particularly problematic by study participants, who felt under extreme pressure to discharge patients quickly so beds were available for new admissions. This pressure led to poor practices, such as foundation doctors writing discharge scripts for patients with whom they had not been involved previously during the admission, and multiple discharge letters being written simultaneously. Prescriptions were written quickly and hampered by interruptions. Problems working out which drugs were needed at discharge also highlighted poor use of medicines reconciliation at admission, and poor documentation of prescribing decisions during patient stay.

Another notable finding was the lack of time foundation doctors perceived they had to check their work, and to return to uncompleted tasks. Attitudes towards prescribing may also be unhelpful, whether perceiving that this is a low risk task or that doctors are merely transcribing information onto drug charts. Foundation doctors were clearly uncomfortable 
about questioning or changing prescriptions made by other team members, either because they assumed they had less experience or because this was against the hierarchical culture. This is in line with Lewis and Tully's recent findings which showed that the multi-disciplinary team and senior doctors in particular, had a strong influence on doctor's prescribing decisions (13), and highlights the importance of healthcare culture which has been rarely studied with reference to prescribing. In addition, foundation doctors believed that errors would be intercepted, and relied heavily on pharmacists checking drug charts. This is of concern, particularly as the study hospitals reported difficulties maintaining a clinical pharmacy service for all wards.

In general, our findings are consistent with similar studies in other countries. It is interesting to note that some findings from other studies did not emerge in this study, such as individual issues of mental and physical health, or a lack of supervision (14). It is not clear whether doctors did not feel these were issues, failed to comment on them or whether this reflects our sample size. It is interesting to note that senior medical supervision of prescribing was only very rarely discussed, which could be interpreted as a lack of supervision being available, or simply that this was not perceived to be a contributing factor.

The study has both strengths and limitations. The study was undertaken in a range of hospital types (locations, sizes), and wards, and included a range of error types. It should therefore be generalisable across NHS Scotland, and given its concurrence with other published work, the rest of the UK. The use of specific occurrences, rather than views on errors in general, is a further strength of the study, as is the timing of the interviews close to the event under study. Possible study limitations include the difficulties in identifying and approaching all doctors who had made an error. Our original intent was to use purposive sampling to select interviewees, but the conditions of Ethical Approval precluded this and required the first approach to be made by the ward pharmacists on our behalf. The ward pharmacists reported informally that they were uncomfortable with our request; they felt that doctors would perceive this approach as negative feedback on their performance and would tarnish their relationship with prescribers. Therefore, all eligible participants were not approached. Furthermore, at times it was difficult to conduct interviews with those doctors who had agreed to participate within the stipulated 96 hours timeframe of the error occurring. Even within this timeframe some prescribers had difficulties remembering specific incidents, attributed primarily to their heavy workload. The relatively small sample size is also a limitation, although the proportion of FY1 to FY2 doctors reflects overall prescribing in the larger study. A further limitation is the possible skewing caused by the 16 errors associated with one patient where the drug chart had the wrong patient name. This study has clarified the latent and error-producing conditions which are perceived to be important by foundation doctors when prescribing. However it is well recognised that perceived causes (attributions) may be subject to systematic bias, minimising participants own responsibilities and emphasising situational factors (15), and this is another potential limitation of this study. 
This study supports the findings of other recent studies $(5,8,11,16)$ in a different geographical location. Whilst many of these issues raised are similar to those reported in the literature, there are some new findings, such as specific issues relating to discharge prescriptions, which are particularly relevant to junior doctor prescribers. Moreover, although our wider study (6) has noted similar rates of error between grades of doctor, it is well accepted that the majority of prescriptions are written by foundation doctors. It is therefore critical to address the particular problems reported by this group in order to have an impact on overall error rates.

This study has emphasised the complex nature of prescribing errors, and the wide range of error-producing conditions within hospitals. Problems were identified in the work environment, team, task, individual and patient. Interventions are needed across these areas, but work environment seems to be critical and may require change at high levels. There is now a critical mass of data to aid the design of interventions to address single or multiple issues. Team interventions are an attractive option and may be beneficial. Individual knowledge is being addressed by various initiatives in undergraduate and postgraduate education such as the UK Prescribing Skills Assessment, but behaviour change techniques may also be useful in addressing individual issues in an educational context. Further work is now needed to develop and assess interventions that address these causes in order to reduce prescribing error rates. 


\section{Tables}

Table 1 Errors by type

\begin{tabular}{|l|l|}
\hline Type of error & $\mathbf{n}(\%)$ \\
\hline Medication omitted & $24(24)$ \\
\hline Wrong patient & $16(16)$ \\
\hline Incorrect dose & $15(15)$ \\
\hline Incorrect frequency & $12(12.2)$ \\
\hline Incorrect timing & $9(9)$ \\
\hline Incomplete prescription & $6(6)$ \\
\hline Incorrect drug & $4(4)$ \\
\hline Incorrect route & $1(1.0)$ \\
\hline Incorrect formulation & $3(3)$ \\
\hline Medication prescribed without indication & $3(3)$ \\
\hline Contra-indication to medication & $3(3)$ \\
\hline Significant drug-drug interaction & $2(2)$ \\
\hline Incorrect duration & $1(1.0)$ \\
\hline Duplication of therapy & $1(1.0)$ \\
\hline Total & $100(100.0)$ \\
\hline
\end{tabular}


Table 2 Errors by Human Error Theory Classification

\begin{tabular}{|l|l|}
\hline Error & Frequency, $\mathbf{n}(\%)$ \\
\hline Slip & $30(30)$ \\
\hline Lapse & $11(11)$ \\
\hline Mistake & $18(18)$ \\
\hline Violation & $6(6)$ \\
\hline Unable to code & $35(35)$ \\
\hline & \\
\hline Total & $100(100)$ \\
\hline
\end{tabular}




\section{Acknowledgements}

The project was funded by the Chief Scientist Office of the Scottish Health Directorates as part of a wider programme of work, the PROTECT Study, investigating the prevalence and causes of errors made by junior doctors. We thank all the hospitals and foundation doctors who took part in the study.

\section{Contributorship statement}

$S R, J F, M J, P D, J K, A L, M M, S M, G M, J M, D W$ and $C B$ were involved in the inception and design of the overall study for which $C B$ was the Chief Investigator, and the other authors are grantholders. SR, CR, ED, JF, MJ and CB designed the interview schedule. CR conducted the interviews. SR and CR analysed the interviews with input from ED, JF and MJ. SR wrote the first draft of the paper, with $C R$ and $C B$. All authors critically reviewed and contributed to the final draft.

\section{Competing interests}

The authors declare that they have no competing interests.

\section{Funding}

Funded by the Chief Scientist Office of the Scottish Health Directorates. 


\section{References}

1. Audit Commission. A spoonful of sugar. Medicines management in NHS hospitals. London: Audit Commission; 2001.

2. NPSA. Safety in doses: improving the use of medicines in the UK. 2007 [cited 29 Feb 2012]. Available from: http://www.nrls.npsa.nhs.uk/resources/?entryid45=61625

3. Pirmohamed $M$, James $S$, Meakin $S$, et al. Adverse drug reactions as cause of admission to hospital: prospective analysis of 18820 patients. BMJ. 2004;329:15-9.

4. Barber N, Rawlins M, Franklin BD. Reducing prescribing error: competence, control, and culture. Qual Saf Health Care 2003;12:29-32.

5. Dornan T, Ashcroft D, Heathfield H, et al. An in-depth investigation into causes of prescribing errors by foundation trainees in relation to their medical education: EQUIP study. Final report to the General Medical Council. University of Manchester: School of Pharmacy and Pharmaceutical Sciences and School of Medicine. 2009 [cited 29 Feb 2012]. Available from:

http://www.pharmacy.manchester.ac.uk/cip/CIPPublications/commissionedreports/prescri bing errors prevalence incidence.pdf

6. Ryan C, Davey P, Francis J, et al. The prevalence of prescribing errors amongst junior doctors in Scotland. Basic Clin Pharmacol Toxicol 2011; 109(1):35.

7. Scottish patient safety programme [cited 29 Feb 2012]. Available from http://www.scottishpatientsafetyprogramme.scot.nhs.uk/programme

8. Dean B, Schachter M, Vincent $C$, et al. Causes of prescribing errors in hospital patients: A prospective study. Lancet 2002;359:1373-8.

9. Dean B, Barber N, Schachter M: What is a prescribing error? Qual Health Care 2000;9:232-7.

10. Ross $\mathrm{S}$, Bond $\mathrm{C}$, Rothnie $\mathrm{H}$, et al. What is the scale of prescribing errors committed by junior doctors? A systematic review. Br J Clin Pharmacol 2009;67(6):629-40.

11. Tully MP, Ashcroft DM, Dornan T, et al. The causes of and factors associated with prescribing errors in hospital inpatients: a systematic review. Drug Saf 2009;32(10):819-36.

12. Reason J. Human error. Cambridge: University of Cambridge, 1990.

13. Lewis $P$, Tully $M$. Uncomfortable prescribing decisions in hospitals: the impact of teamwork. J R Soc Med 2009;102:481-8. 
14. Coombes I, Stowasser D, Coombes J, et al. Why do interns make prescribing errors? Implications for junior doctor supervision, training and other strategies to improve medication safety. Med J Aust 2008;188:89-94.

15. Campbell WK, Sedikides C. Self-threat magnifies the self-serving bias: A meta-analytic integration. Rev Gen Psychol 1999;3:23-43.

16. Franklin BD, Reynolds M, Shebl NA, Burnett S, Jacklin A. Prescribing errors in hospital inpatients: a three-centre study of their prevalence, types and causes. Postgrad Med J 2011;87:739-745. 